astro-ph/0408278, INJE-TP-04-06

\title{
Cosmological constraints on inflation potential: the role of Gauss-Bonnet corrections in braneworld scenarios
}

\author{
Kyong Hee Kim and Yun Soo Myung* \\ Relativity Research Center and School of Computer Aided Science \\ Inje University, Gimhae 621-749, Korea
}

\begin{abstract}
We calculate the spectral index and tensor-to-scalar ratio for patch inflation defined by $H^{2} \approx \beta_{q}^{2} V^{q}$ and $\dot{\phi} \approx-V^{\prime} / 3 H$, using the slow-roll expansion. The patch cosmology arisen from the Gauss-Bonnet braneworld consists of Gauss-Bonnet (GB), Randall-Sundrum (RS), and 4D general relativistic (GR) cosmological models. In this work, we choose large-field potentials of $V=$ $V_{0} \phi^{p}$ to compare with the observational data. Since second-order corrections are rather small in the slow-roll limit, the leading-order calculation is sufficient to compare with the data. Finally, we show that it is easier to discriminate between quadratic potential and quartic potential in the GB cosmological model rather than the GR or RS cosmological models.
\end{abstract}

*E-mail address: ysmyung@physics.inje.ac.kr 


\section{INTRODUCTION}

There has been much interest in the phenomenon of localization of gravity proposed by Randall and Sundrum (RS) [1]. They assumed a 3-brane with a positive-tension in the five dimensional (5D) anti-de Sitter spacetime. They obtained a localized gravity on the brane by fine-tuning the tension to the cosmological constant. Recently, several authors studied the cosmological implications of a brane world scenario. The brane cosmology contains some important deviations from the Friedmann-Robertson-Walker (FRW) cosmology [2,3]. Especially, the Friedmann equation is modified in the high-energy regime significantly.

On the other hand, it is generally accepted that curvature perturbations produced during inflation are considered to be the origin of inhomogeneities necessary for explaining cosmic microwave background (CMB) anisotropies and large-scale structures. The latest results come from Wilkinson Microwave Anisotropy Probe (WMAP) [4], Sloan Digital Sky Survey (SDSS) [5] and others. These put forward more constraints on cosmological models and confirm the emerging standard model of cosmology, a flat $\Lambda$-dominated universe seeded by scale-invariant adiabatic Gaussian fluctuations. In other words, these results coincide with theoretical predictions of the slow-roll inflation based on general relativity with a single inflaton.

If the brane inflation occurs, one expects that it provides us different results in the highenergy regime [6-10]. In the slow-roll approximation $[11,12]$, there is no significant change in the power spectrum between the standard and brane cosmology up to first-order corrections [13]. In order to distinguish between the standard and brane inflation apparently, it is necessary to calculate their power spectra up to second-order using the slow-roll expansion [14]. Unfortunately, it is not easy to discriminate between the standard and brane inflation because second-order corrections are rather small in the slow-roll limit [15]. Furthermore, the degeneracy exists between scalar and tensor perturbation. This is expressed as the consistency relation of $R=-8 n_{T}$ in the standard inflation. This relation remains unchanged even in the brane cosmology $[16,17]$. In order to handle this degeneracy problem, the authors in [18] calculated the tensor spectrum in the Gauss-Bonnet braneworld. They found that this relation could be broken by the Gauss-Bonnet term. However, this breaking is so mild that the likelihood values for the Gauss-Bonnet brane inflation are nearly identical to those in the standard inflation [19]. Thus it seems that the introduction of a Gauss-Bonnet term in the braneworld could not distinguish between the standard inflation and brane inflation.

In this work, we study whether or not the quartic potential $\left(V=V_{0} \phi^{4}\right)$ could be ruled out in view of the patch inflation defined by $H^{2} \approx \beta_{q}^{2} V^{q}, \dot{\phi} \approx-V^{\prime} / 3 H$. We note that the patch cosmology comes from the Gauss-Bonnet braneworld. First, in order to see the difference between different models, we calculate the scalar spectral index up to second-order using the slow-roll expansion. Since second-order corrections are rather small in the slow-roll limit, their theoretical points are not significantly moved from the leading-order results. Thus we use the leading-order spectral index and tensor-to-scalar ratio to select which model is mostly suitable for discriminating between quadratic potential and quartic potential. As a result, the GB model is turned out to be the best one to choose a favorable potential of $V=V_{0} \phi^{2}$ and to reject an unfavorable potential of $V=V_{0} \phi^{4}$.

The organization of this paper is as follows. In Section II we briefly review the patch cosmology and relevant cosmological parameters. We choose large-field potentials to compare 
TABLE I. Three relevant models for the patch cosmology and their parameters classifying different models.

\begin{tabular}{lll}
\hline \hline model & $q(\theta)$ & $\beta_{q}^{2}$ \\
\hline GB & $2 / 3(-1)$ & $\left(\kappa_{5}^{2} / 16 \alpha\right)^{2 / 3}$ \\
RS & $2(1)$ & $\kappa_{4}^{2} / 6 \lambda$ \\
GR & $1(0)$ & $\kappa_{4}^{2} / 3$ \\
\hline \hline
\end{tabular}

the theoretical points with the observation data in Section III. Finally we discuss our results in Section IV. We list the second-order corrections to cosmological parameters in Appendix A and their explicit forms for large-field potentials in Appendix B.

\section{PATCH COSMOLOGY}

We start with an effective Friedmann equation arisen from the Gauss-Bonnet braneworld by adopting a flat FRW metric as the background spacetime on the brane ${ }^{1}[13,18,19]$

$$
H^{2}=\beta_{q}^{2} \rho^{q},
$$

where $H=\dot{a} / a$ is the Hubble parameter, $q$ is a parameter describing each cosmological model, and $\beta_{q}^{2}$ is a factor with energy dimension $\left[\beta_{q}\right]=E^{1-2 q}$. An additional parameter defined by $\theta=2(1-1 / q)$ is introduced for convenience. We call the above defined on the $q$-dependent energy regimes as a whole "patch cosmology". We summarize relevant cosmological models and their parameters in the patch cosmology in Table I. $\kappa_{5}^{2}=8 \pi M_{5}^{-3}\left(\kappa_{4}^{2}=8 \pi M_{4}^{-2}\right)$ is the 5D (4D) gravitational coupling constant. $\alpha$ is the GaussBonnet coupling which may be related to the string energy scale $g_{s}$ as $\alpha=1 / 8 g_{s}$ and $\lambda$ is a brane tension. The two relations between these are $\kappa_{4}^{2} / \kappa_{5}^{2}=\mu /(1+\beta)$ and $\lambda=2 \mu(3-\beta) / \kappa_{5}^{2}$, where $\beta=4 \alpha \mu^{2} \ll 1, \mu=1 / \ell$ with an $\mathrm{AdS}_{5}$ curvature radius $\ell$. The Randall-Sundrum case of $\mu=\kappa_{4}^{2} / \kappa_{5}^{2}$ is recovered when $\alpha=0$. Introducing an inflaton $\phi$ confined to the brane, one finds the equation

$$
\ddot{\phi}+3 H \dot{\phi}=-V^{\prime}
$$

where the dot and prime denote the derivative with respect to time $t$ and $\phi$, respectively. Its energy density and pressure are given by $\rho=\dot{\phi} / 2+V$ and $p=\dot{\phi} / 2-V$. From now on we use the slow-roll formalism for inflation: an accelerated universe $(\ddot{a}>0)$ is being driven by a single scalar field slowly rolling down its potential toward a local minimum. This means that Eqs.(1) and (2) take the approximate forms:

\footnotetext{
${ }^{1}$ For reference, we add the action for the Gauss-Bonnet braneworld scenario [8]: $S=$ $\frac{1}{2 \kappa_{5}^{2}} \int_{\text {bulk }} d^{5} x \sqrt{-g_{5}}\left[R-2 \Lambda_{5}\left(R^{2}-4 R_{\mu \nu} R^{\mu \nu}+R_{\mu \nu \rho \sigma} R^{\mu \nu \rho \sigma}\right)\right]+\int_{\text {brane }} d^{4} x \sqrt{-g}\left[-\lambda+\mathcal{L}_{\text {matter }}\right]$ with $\Lambda_{5}=-3 \mu^{2}(2-\beta)$ for an AdS bulk cosmological constant and $\mathcal{L}_{\text {matter }}$ for inflation on the brane. Its full Friedmann equation is given by a complicated form $2 \mu\left(1+H^{2} / \mu^{2}\right)^{1 / 2}\left[3-\beta+3 \beta H^{2} / \mu^{2}\right]=$ $\kappa_{5}^{2}(\rho+\lambda)$.
} 


$$
H^{2} \approx \beta_{q}^{2} V^{q}, \dot{\phi} \approx-V^{\prime} / 3 H
$$

This implies that the cosmological acceleration is given by a fluid with a vacuum-like equation of state $p \approx-\rho$. If $p=-\rho$ with $\dot{\phi}=0$, it corresponds to a de Sitter inflation with $a(t)=a_{0} e^{H t}$. We note that this is the only background to obtain gravitational waves from the braneworld scenario [20]. In order for an inflation to end and the universe to transfer to the radiation-dominated universe, we need the slow-roll mechanism. For this purpose, we introduce Hubble slow-roll parameters $\left(\epsilon_{1}, \delta_{n}\right)$ on the brane as

$$
\epsilon_{1} \equiv-\frac{\dot{H}}{H^{2}} \approx \epsilon_{1}^{q} \equiv \frac{q}{6 \beta_{q}^{2}} \frac{V^{\prime 2}}{V^{1+q}}, \quad \delta_{n} \equiv \frac{1}{H^{n} \dot{\phi}} \frac{d^{n+1} \phi}{d t^{n+1}} \approx \delta_{n}^{q},
$$

where they satisfy the slow-roll condition: $\epsilon_{1}<\xi,\left|\delta_{n}\right|<\xi^{n}$ for a small parameter $\xi$. Here the subscript denotes slow-roll (SR)-order in the slow-roll calculation. A slow-roll parameter $\epsilon_{1}$ controls the equation of states $p=\left(-1+2 \epsilon_{1} / 3\right) \rho$ microscopically, which implies that an accelerating expansion is always possible for $\epsilon_{1}<1(p<-\rho / 3)$ [21]. The case of $\epsilon_{1}=0$ corresponds to a de Sitter inflation. If one chooses the inflation potential $V$ explicitly, potential slow-roll parameters $\left(\epsilon_{1}^{q}, \delta_{n}^{q}\right)$ will be found.

We review how to calculate the cosmological parameters using the slow-roll formalism. Introducing a new variable $u^{q}=a\left(\delta \phi^{q}-\dot{\phi} \psi^{q} / H\right)$ where $\delta \phi^{q}\left(\psi^{q}\right)$ is a perturbed inflaton (perturbed metric), its Fourier modes $u_{k}^{q}$ in the linear perturbation theory satisfies the Mukhanov -type equation ${ }^{2}[22]$ :

$$
\frac{d^{2} u_{k}^{q}}{d \tau^{2}}+\left(k^{2}-\frac{1}{z_{q}} \frac{d^{2} z_{q}}{d \tau^{2}}\right) u_{k}^{q}=0 .
$$

Here $\tau$ is the conformal time defined by $d \tau=d t / a$ and $z_{q}=a \dot{\phi} / H$ is a parameter encoded all information about a slow-roll inflation.

Before we proceed, we have to mention that Eq.(5) is the nearly same form as in the conventional 4D perturbation theory [22]. It is well known that the perturbation theory of braneworlds including Randall-Sundrum and Gauss-Bonnet models is very different from the 4D-GR perturbation theory $[6,7,9,10,16,18,20]$. Making use of the 4D Mukhanov equation to study the braneworld perturbation, the problem is that this equation incorporates $4 \mathrm{D}$ metric (scalar) perturbations only and thus there is no justification for using this to describe the effect of 5D gravity on the brane. This falls short of being a full 5D calculation as is required by the braneworld sceranio. However, it was shown recently that even though the effect of 5D metric perturbations on inflation appears to be large on small-scales (subhorizon), on large-scales (super-horizon) this effect is smaller than slow-roll corrections to de Sitter background [23]. Further, the effect of 5D metric perturbations is very small, at

\footnotetext{
${ }^{2}$ We list the $q$-dependent potential: $\frac{1}{z_{q}} \frac{d^{2} z_{q}}{d \tau^{2}}=2 a^{2} H^{2}\left(1+\epsilon_{1}+\frac{3}{2} \delta_{1}+\frac{1}{q} \epsilon_{1}^{2}+2 \epsilon_{1} \delta_{1}+\frac{1}{2} \delta_{2}\right)$. Here one change occurs in coefficient of $\epsilon_{1}^{2}: 1 \rightarrow 1 / q$. Although the Gauss-Bonnet brane cosmology provides a complicated form, its patch approximation provides simple $q$-potentials with minor change. This is the main reason why we choose the patch cosmology instead of the full Gauss-Bonnet brane cosmology.
} 
low energies, on super-horizon and also this is suppressed, even at high energies, on superhorizon. Therefore it is sensible to use the 4D perturbation theory, especially for the study of GB and RS models in the high-energy regime because we always compute the cosmological parameters on the super-horizon scale.

The asymptotic solutions to Eq.(5) are obtained as

$$
u_{k}^{q} \longrightarrow \begin{cases}\frac{1}{\sqrt{2 k}} e^{-i k \tau} & \text { as }-k \tau \rightarrow \infty \\ \mathcal{C}_{k}^{q} z_{q} & \text { as }-k \tau \rightarrow 0 .\end{cases}
$$

The first solution corresponds to a plane wave on scale much smaller than the Hubble horizon of $d_{H}=1 / H$ (sub-horizon), while the second is a growing mode on scale much larger than the Hubble horizon (super-horizon). Using a relation of $R_{c \mathbf{k}}^{q}=-u_{\mathbf{k}}^{q} / z_{q}$ with $u_{\mathbf{k}}^{q}(\tau)=a_{\mathbf{k}} u_{k}^{q}(\tau)+a_{-\mathbf{k}}^{\dagger} u_{k}^{q *}(\tau)$ and a definition of $P_{R_{c}}^{q}(k) \delta^{(3)}(\mathbf{k}-\mathbf{l})=\frac{k^{3}}{2 \pi^{2}}<R_{c \mathbf{k}}^{q}(\tau) R_{c \mathbf{l}}^{q \dagger}(\tau)>$, one finds the power spectrum for a curvature perturbation on the super-horizon scale

$$
P_{R_{c}}^{q}(k)=\left(\frac{k^{3}}{2 \pi^{2}}\right) \lim _{-k \tau \rightarrow 0}\left|\frac{u_{k}^{q}}{z_{q}}\right|^{2}=\frac{k^{3}}{2 \pi^{2}}\left|\mathcal{C}_{k}^{q}\right|^{2} .
$$

Our task is to find $\mathcal{C}_{k}^{q}$ by solving the Mukhanov-type equation (5). In general, it is not easy to find a solution to this equation. However, we could solve it by using either the slow-roll approximation [11] or the slow-roll expansion [14]. In the slow-roll approximation we take $\epsilon_{1}$ and $\delta_{1}$ to be constant. Thus this method could not be considered as a general approach beyond the first-order correction to the power spectrum [24,25]. In order to calculate the power spectrum up to second-order, one uses the slow-roll expansion based on Green's function technique. A key step is to account for a slowly varying nature of slow-roll parameters implied by two equations $\dot{H}=-\frac{3}{2} q \beta_{q}^{2} \rho^{q-1} \dot{\phi}^{2}$ and $\ddot{H}=2 H \dot{H}\left[-(1-1 / q) \epsilon_{1}+\delta_{1}\right]$ :

$$
\begin{aligned}
& \dot{\epsilon}_{1}=2 H\left(\epsilon_{1}^{2} / q+\epsilon_{1} \delta_{1}\right), \dot{\delta}_{1}=H\left(\epsilon_{1} \delta_{1}-\delta_{1}^{2}+\delta_{2}\right), \\
& \dot{\delta}_{2}=H\left(2 \epsilon_{1} \delta_{2}-\delta_{1} \delta_{2}+\delta_{3}\right), \dot{\delta}_{3}=H\left(3 \epsilon_{1} \delta_{3}-\delta_{1} \delta_{3}+\delta_{4}\right) .
\end{aligned}
$$

The above means that the derivative of slow-roll parameters with respect to time increases their SR-order by one. After a lengthly calculation following Ref. [14,26], we obtain the $q$ power spectrum, $q$-spectral index, and $q$-running spectral index. See Appendix A, for their explicit forms.

The tensor-to-scalar ratio $R_{q}$ is defined by

$$
R_{q}=16 \frac{A_{T, q}^{2}}{A_{S, q}^{2}} .
$$

Here the $q$-scalar amplitude in the leading-order is normalized by

$$
A_{S, q}^{2}=\frac{4}{25} P_{R_{c}}^{q, E S R}
$$

with the extreme slow-roll (ESR) power spectrum ${ }^{3}$

\footnotetext{
${ }^{3}$ Here, the extreme slow-roll limit means that $\epsilon_{1} \rightarrow 0$ and $\delta_{n} \rightarrow 0$ in Eq.(18). Actually, this corresponds to the background spacetime of de Sitter inflation with $H=$ const for the slow-roll expansion.
} 


$$
P_{R_{c}}^{q, E S R}=\frac{3 q \beta_{q}^{2-\theta}}{(2 \pi)^{2}} \frac{H^{2+\theta}}{2 \epsilon_{1}}=\frac{1}{(2 \pi)^{2}} \frac{H^{4}}{\dot{\phi}^{2}} .
$$

The $\operatorname{GR}(q=1)$ tensor amplitude up to leading-order is given by

$$
A_{T, G R}^{2}=\frac{1}{50} P_{T}^{E S R}
$$

where $P_{T}^{E S R}=\left(2 \kappa_{4}\right)^{2}\left(\frac{H}{2 \pi}\right)^{2}$ since a tensor can be expressed in terms of two scalars like $\delta \phi$. For the GR case, we could calculate tensor spectra up to higher-order as we wish to do using the slow-roll expansion. On the other hand, the tensor spectra for GB and RS models are known only for de Sitter brane like $a(t) \sim e^{H t}[20,18]$. This means that tensor computation should be limited to the leading-order in our calculation. These are given by

$$
A_{T, q}^{2}=A_{T, G R}^{2} F_{\beta}^{2}(H / \mu),
$$

where

$$
F_{\beta}^{-2}(x)=\sqrt{1+x^{2}}-\left(\frac{1-\beta}{1+\beta}\right) x^{2} \sinh ^{-1}\left(\frac{1}{x}\right)
$$

In three different regimes, we approximate $F_{\beta}^{2}$ as $F_{q}^{2}: F_{1}^{2} \approx F_{\beta}^{2}(H / \mu \ll 1)=1$ for the GR case ; $F_{2}^{2} \approx F_{\beta=0}^{2}(H / \mu \gg 1)=3 H /(2 \mu)$ for the RS case ; $F_{2 / 3}^{2} \approx F_{\beta}^{2}(H / \mu \gg 1)=$ $(1+\beta) /(2 \beta)(\mu / H)$ for the GB case. The $q$-tensor amplitude up to leading-order is given by

$$
A_{T, q}^{2}=\frac{3 q \beta_{q}^{2-\theta}}{(5 \pi)^{2}} \frac{H^{2+\theta}}{2 \zeta_{q}}
$$

with $\zeta_{1}=\zeta_{2 / 3}=1$ and $\zeta_{2}=2 / 3[27]$. Finally, the tensor-to-scalar ratio is determined by

$$
R_{q}=16 \frac{A_{T, q}^{2}}{A_{S, q}^{2}}=16 \frac{\epsilon_{1}}{\zeta_{q}} .
$$

Considering a relation of $n_{T}^{q}=-(2+\theta) \epsilon_{1}$, one finds that

$$
R_{1}=-8 n_{T}^{1}=16 \epsilon_{1}, R_{2}=-8 n_{T}^{2}=24 \epsilon_{1}, R_{2 / 3}=-16 n_{T}^{2 / 3}=16 \epsilon_{1} .
$$

The above shows that the RS-consistency relation takes the same form for GR case, but the GB-consistency relation is different from those of RS and GR cosmological models.

\section{INFLATION WITH LARGE-FIELD POTENTIALS}

First we calculate the scalar cosmological parameters for the large-field model using slow-roll expansion because there is no upper-limit on this calculation. We choose largefield potentials (LF) like $V(\phi)=V_{0} \phi^{p}$ with $p=2,4,6$ for our purpose. The LF-slow-roll parameters (V-SR), LF-power spectrum, LF-spectral index, and LF-running spectral index are given by Appendix B. According to Ref. [28], the quartic potential is observationally disfavored when using the GR model because the theoretical points are outside the $2 \sigma$ 
TABLE II. The higher-order corrections to the spectral index $\left(n_{s}\right)$ and running spectral index $\left(\alpha \equiv d n_{s} / d \ln k\right)$. Here we choose $N=50$ to find all theoretical parameters for the large-field potentials (LF). For GB model with $p=6$, we have to use the other result such as the power-law inflation in Table III.

\begin{tabular}{|c|c|c|c|c|}
\hline Patch & $p$ & Leading-order & First-order & Second-order \\
\hline \multirow{3}{*}{$\begin{array}{c}\mathrm{GB} \\
(q=2 / 3)\end{array}$} & 2 & $\begin{array}{c}n_{s}=0.9703 \\
\alpha=-0.0006\end{array}$ & \begin{tabular}{c|}
0.9700 \\
-0.0006
\end{tabular} & $\begin{array}{c}0.9700 \\
-0.0006\end{array}$ \\
\hline & 4 & $\begin{array}{c}n_{s}=0.9423 \\
\alpha=-0.0011\end{array}$ & $\begin{array}{c}0.9393 \\
-0.0013 \\
\end{array}$ & $\begin{array}{c}0.9392 \\
-0.0013 \\
\end{array}$ \\
\hline & 6 & $\begin{array}{l}\mathrm{N} / \mathrm{A} \\
\mathrm{N} / \mathrm{A}\end{array}$ & $\begin{array}{l}\mathrm{N} / \mathrm{A} \\
\mathrm{N} / \mathrm{A}\end{array}$ & $\begin{array}{l}\mathrm{N} / \mathrm{A} \\
\mathrm{N} / \mathrm{A}\end{array}$ \\
\hline \multirow{3}{*}{$\begin{array}{c}\mathrm{GR} \\
(q=1)\end{array}$} & 2 & $\begin{array}{c}n_{s}=0.9604 \\
\alpha=-0.0008\end{array}$ & $\begin{array}{c}0.9602 \\
-0.0008 \\
\end{array}$ & $\begin{array}{c}0.9602 \\
-0.0008 \\
\end{array}$ \\
\hline & 4 & $\begin{array}{c}n_{s}=0.9412 \\
\alpha=-0.0012\end{array}$ & $\begin{array}{c}0.9401 \\
-0.0012 \\
\end{array}$ & $\begin{array}{c}0.9401 \\
-0.0012 \\
\end{array}$ \\
\hline & 6 & $\begin{array}{c}n_{s}=0.9223 \\
\alpha=-0.0015\end{array}$ & $\begin{array}{c}0.9200 \\
-0.0016\end{array}$ & $\begin{array}{c}0.9200 \\
-0.0016\end{array}$ \\
\hline \multirow{3}{*}{$\begin{array}{c}\mathrm{RS} \\
(q=2)\end{array}$} & 2 & $\begin{array}{c}n_{s}=0.9505 \\
\alpha=-0.001\end{array}$ & $\begin{array}{r}0.9503 \\
-0.001 \\
\end{array}$ & $\begin{array}{l}0.9503 \\
-0.001 \\
\end{array}$ \\
\hline & 4 & $\begin{array}{c}n_{s}=0.9408 \\
\alpha=-0.0012\end{array}$ & $\begin{array}{c}0.9403 \\
-0.0012 \\
\end{array}$ & $\begin{array}{c}0.9404 \\
-0.0012 \\
\end{array}$ \\
\hline & 6 & $\begin{array}{c}n_{s}=0.9360 \\
\alpha=-0.0013\end{array}$ & $\begin{array}{c}0.9354 \\
-0.0013\end{array}$ & $\begin{array}{c}0.9354 \\
-0.0013\end{array}$ \\
\hline
\end{tabular}

contour bound for $45 \leq N<60$. We note that a range of $50 \leq N \leq 60$ is usually used for a typical calculation of inflation. The potentials of $V=V_{0} \phi^{p}$ with $p \geq 6$ are ruled out by the CMB alone [5]. A potential of $V=V_{0} \phi^{2}$ is considered as a promising one because it is inside the $1 \sigma$ bound for $50 \leq N \leq 60$. For the RS model, the quartic potential is under strong observational pressure as is similar to the GR case because it is outside the $2 \sigma$ bound for $45 \leq N \leq 60$. The quadratic potential in the RS model is inside the $2 \sigma$ bound for $50 \leq N \leq 60$. The exponential potential is ruled out for the RS case since it is far outside the $2 \sigma$ bound. The quartic potential is ruled out for the GB model because it is far outside the $2 \sigma$ bound, while the quadratic potential is inside the $1 \sigma$ bound for $45 \leq N \leq 60$. It implies that $V=V_{0} \phi^{2}$ is considered as a promising potential for GR and GB models, while $V=V_{0} \phi^{4}$ is under significant pressure from data. However, it does not mean that the quartic potential is ruled out by the current observation completely.

In this section we investigate this problem more carefully. We observe from Table II that the theoretical parameters are not significantly changed by the SR higher-order calculations. In other words, the theoretical parameters are insensitive to the higher-order corrections. It confirms that the leading-order calculation is sufficient to compare with the observation data in the slow-roll limit. All of the running spectral indices are very small when comparing with the WMAP data of $d n_{s} / d \ln k=-0.031_{-0.018}^{+0.016}$ at $k_{0}=0.05 \mathrm{Mpc}^{-1}$ [4]. Hence the large-field model is close to the vanilla model with the zero-running spectral index [5].

On the other hand, the theoretical parameters are significantly changed by introducing 
TABLE III. Potentials, spectral index, running spectral index, and tensor-to-scalar ratio for a power-law inflation with $a(t)=a_{0} t^{r}$ with $r>1$. Three different potentials give the nearly same result when the patch cosmology is applied to calculate cosmological parameters.

\begin{tabular}{c|c|c|c|c}
\hline \hline model & $V^{P I}$ & $d n_{s}^{P I}$ & $d n_{s}^{P I} / d \ln k$ & $R^{P I}$ \\
\hline GB & $V_{0} \phi^{6}$ & $1-\left[\frac{2 r-1}{2 r^{4}}\right]^{\frac{1}{3}}-\left[\frac{2 r-1}{2 r^{4}}\right]^{\frac{2}{3}}-\left[\frac{2 r-1}{2 r^{4}}\right]$ & 0 & $16 / r$ \\
GR & $V_{0} \exp \left(-\sqrt{2 \kappa_{4}^{2} / r} \phi\right)$ & $1-\frac{2}{r}-\frac{2}{r^{2}}-\frac{2}{r^{3}}$ & 0 & $16 / r$ \\
RS & $V_{0} \phi^{-2}$ & $1-3\left[\frac{6}{6 r-1}\right]-3\left[\frac{6}{6 r-1}\right]^{2}-3\left[\frac{6}{6 r-1}\right]^{3}$ & 0 & $24 / r$ \\
\hline \hline
\end{tabular}

different cosmological models. In testing the large-field model with the data, a significant difference appears between GB, GR and RS models. Here we mainly use the two important parameters: the spectral index $n_{s}^{q}$ and $R_{q}$ obtained from the leading-order calculation: the first line in Eq.(24) and Eq.(16) together with $\epsilon_{1}^{q}$ in Eq.(22). What we want to do is to find which $q$-model moves theoretical points predicted by a given potential quickly inside the $1 \sigma$ bound contour $\left(n_{s}^{q} \rightarrow 1, R_{q} \rightarrow 0\right.$ and $d n_{s} / d \ln k \rightarrow 0$ inspired by the vanilla model) for $50 \leq N \leq 60$.

Consequently, the GB model is regarded as a promising one because it accepts the quadratic potential clearly, but it rejects the quartic potential because theoretical points are far outside the $2 \sigma$ bound. The GB model improves the theoretical values predicted by the GR case, whereas the RS model provides indistinctive values more than the GR case. Actually the GB model splits large-field potentials into three distinct regions clearly: for $N=50, n_{s}^{G B}=0.97 \rightarrow 0.94(p=2 \rightarrow p=4), R_{G B}=0.16 \rightarrow 0.61$ and the powerlaw inflation with $p=6: n_{s}^{P I}=1-\left[(2 r-1) / 2 r^{4}\right]^{1 / 3}, R_{P I}=16 / r$ (see Table III). Here, for the GB model with $p=6$, we have to use the result of the power-law inflation which corresponds to the border between large-field and hybrid models. This implies that for the GB case, $p=6$ no longer belong a class of large-field potentials. On the other hand, we have $n_{s}^{G R}=0.96 \rightarrow 0.94 \rightarrow 0.92(p=2 \rightarrow p=4 \rightarrow p=6), R_{G R}=0.16 \rightarrow$ $0.31 \rightarrow 0.47$, whereas $n_{s}^{R S}=0.95 \rightarrow 0.94 \rightarrow 0.94, R_{R S}=0.24 \rightarrow 0.32 \rightarrow 0.35$. For $N=60, n_{s}^{G B}=0.98 \rightarrow 0.95(p=2 \rightarrow p=4), R_{G B}=0.13 \rightarrow 0.52$ and the powerlaw inflation with $p=6$ : $n_{s}^{P I}=1-\left[(2 r-1) / 2 r^{4}\right]^{1 / 3}, R_{P I}=16 / r$. Also we obtain $n_{s}^{G R}=0.97 \rightarrow 0.95 \rightarrow 0.93(p=2 \rightarrow p=4 \rightarrow p=6), R_{G R}=0.13 \rightarrow 0.26 \rightarrow 0.39$, while $n_{s}^{R S}=0.96 \rightarrow 0.95 \rightarrow 0.95, R_{R S}=0.20 \rightarrow 0.26 \rightarrow 0.30$. The theoretical points predicted by the RS model lie very close to the border between the regions allowed and disallowed by the observation.

As is shown in Table III, three different potentials give the nearly same power-law inflation when the patch cosmology is used to calculate the cosmological paramters [26]. We find the allowed steps from the observationally favored potential to the power-law inflation which corresponds to the border between large-field and hybrid models: for the GB case, $\phi^{2} \rightarrow \phi^{4} \rightarrow \phi^{6}$ (three steps), for the GR case, $\phi^{2} \rightarrow \phi^{4} \rightarrow \phi^{6} \rightarrow e^{-\phi}\left(\approx \phi^{p}, p \rightarrow \infty\right)$ (four steps), and for the RS case, $\phi^{2} \rightarrow \phi^{4} \rightarrow \phi^{6} \rightarrow e^{-\phi} \rightarrow \phi^{-2}$ (five steps). 


\section{DISCUSSIONS}

First of all, we mention that Eq.(5) incorporates 4D metric (scalar) perturbations in the cosmological perturbation theory and thus there is no justification for using this to describe the effect of 5D gravity on inflation confined to the brane. However, it was shown recently that at low energies, the effect of 5D metric perturbations is very small on super-horizon and even at high energies, this is suppressed on super-horizon. Hence it is not serious to use the $4 \mathrm{D}$ cosmological perturbation theory, especially for the study of GB and RS cosmological models in the high-energy regime because we always compute the cosmological parameters on the super-horizon scale.

Since second-order corrections are rather small in the slow-roll limit, their theoretical points are not significantly moved from the leading-order results. Hence we use the results from the leading-order calculation to compare the theoretical parameters with the data. It turns out that the GB cosmological model is a promising model to discriminate between the quadratic potential and quartic potential, when comparing with the observation data. This is mainly because the GB model divides large-field potentials into three distinct regions in the $n_{s}^{q}-R_{q}$ plane clearly: $p=2$ (inside the $1 \sigma$ bound), $p=4$ (far outside the $2 \sigma$ bound), $p=6$ ( the border between large-fields and hybrid models). On the other hand, the GR (RS) models require four (five) regions in the $n_{s}^{q}-R_{q}$ plane. The GB model provides the tightest constraint on the large-field model, whereas the RS model provides the loosest constraint because the theoretical points are too close to distinguish between quadratic potential and quartic potential. In this sense, the Randall-Sundrum braneworld in the high-energy regime (the RS model) is regarded to be the worst case.

Consequently, we show that it is easier to discriminate between quadratic and quartic inflation potentials in the GB cosmological model rather than the GR or RS cosmological models.

\section{Acknowledgements}

We thank Hungsoo Kim, H. W. Lee and G. Calcagni for helpful discussions. Y.S. was supported in part by KOSEF, Project No. R02-2002-000-00028-0. K.H. was in part supported by KOSEF, Astrophysical Research Center for the Structure and Evolution of the Cosmos.

\section{Appendix A: Second-order corrections to cosmological parameters}

The $q$-power spectrum is calculated as

$$
\begin{aligned}
P_{R_{c}}^{q}(k)= & \frac{H^{4}}{(2 \pi)^{2} \dot{\phi}^{2}}\left\{1-2 \epsilon_{1}+2 \alpha\left(2 \epsilon_{1}+\delta_{1}\right)\right. \\
& +\left((8-4 / q) \alpha^{2}-4(1-1 / q) \alpha-(19+4 / q)+(2+1 / 3 q) \pi^{2}\right) \epsilon_{1}^{2} \\
& \left.+\left(3 \alpha^{2}+2 \alpha-22+29 \pi^{2} / 12\right) \epsilon_{1} \delta_{1}+\left(3 \alpha^{2}-4+5 \pi^{2} / 12\right) \delta_{1}^{2}-\left(\alpha^{2}-\pi^{2} / 12\right) \delta_{2}\right\}
\end{aligned}
$$


and the right hand side should be evaluated at horizon crossing of $k=a H$. $\alpha$ is defined by $\alpha=2-\ln 2-\gamma \simeq 0.7296$, where $\gamma$ is the Euler-Mascheroni constant with $\gamma \simeq 0.5772$. We note that the $q$-dependent terms appear in the second-order corrections. Using a relation of $d \ln k \simeq H d t$ and Eq.(8), the $q$-spectral index defined by

$$
n_{s}^{q}(k)=1+\frac{d \ln P_{R_{c}}^{q}}{d \ln k}
$$

can be calculated as

$$
\begin{aligned}
n_{s}^{q}(k)= & 1-4 \epsilon_{1}-2 \delta_{1}+(-4-4 / q+8 \alpha / q) \epsilon_{1}^{2}+(10 \alpha-6) \epsilon_{1} \delta_{1}-2 \alpha \delta_{1}^{2}+2 \alpha \delta_{2} \\
& +\left\{-16 \alpha^{2} / q^{2}+\left(16 / q^{2}+24 / q\right) \alpha-4-16 / q^{2}-88 / q+\left(4 / 3 q^{2}+8 / q\right) \pi^{2}\right\} \epsilon_{1}^{3} \\
& +\left\{-(26 / q+5) \alpha^{2}+(32+28 / q) \alpha-112-60 / q+(125 / 12+37 / 6 q) \pi^{2}\right\} \epsilon_{1}^{2} \delta_{1} \\
& +\left(-3 \alpha^{2}+4 \alpha-30+13 \pi^{2} / 4\right) \epsilon_{1} \delta_{1}^{2}+\left(-7 \alpha^{2}+8 \alpha-22+31 \pi^{2} / 12\right) \epsilon_{1} \delta_{2} \\
& +\left(-2 \alpha^{2}+8-5 \pi^{2} / 6\right) \delta_{1}^{3}+\left(3 \alpha^{2}-8+3 \pi^{2} / 4\right) \delta_{1} \delta_{2}+\left(-\alpha^{2}+\pi^{2} / 12\right) \delta_{3} .
\end{aligned}
$$

The $q$-running spectral index is determined by

$$
\begin{aligned}
\frac{d}{d \ln k} n_{s}^{q} & =-8 \epsilon_{1}^{2} / q-10 \epsilon_{1} \delta_{1}+2 \delta_{1}^{2}-2 \delta_{2} \\
& +\left(-16 / q^{2}-24 / q+32 \alpha / q^{2}\right) \epsilon_{1}^{3} \\
& +(-32-28 / q+(10+52 / q) \alpha) \epsilon_{1}^{2} \delta_{1} \\
& +(6 \alpha-4) \epsilon_{1} \delta_{1}^{2}+(14 \alpha-8) \epsilon_{1} \delta_{2}+4 \alpha \delta_{1}^{3}-6 \alpha \delta_{1} \delta_{2}+2 \alpha \delta_{3} \\
& +\left\{-96 \alpha^{2} / q^{2}+\left(96 / q^{3}+176 / q^{2}\right) \alpha-\left(96 / q^{3}+544 / q^{2}+48 / q\right)+\left(8 / q^{3}+48 / q^{2}\right) \pi^{2}\right\} \epsilon_{1}^{4} \\
& +\left\{-\left(200 / q^{2}+46 / q+5\right) \alpha^{2}+\left(208 / q^{2}+352 / q+42\right) \alpha\right\} \epsilon_{1}^{3} \delta_{1} \\
& +\left\{-\left(336 / q^{2}+1064 / q+168\right)+\left(98 / 3 q^{2}+575 / 6 q+125 / 12\right) \pi^{2}\right\} \epsilon_{1}^{3} \delta_{1} \\
& +\left\{-(84 / q+21) \alpha^{2}+(92 / q+100) \alpha-(240 / q+400)+(25 / q+151 / 4) \pi^{2}\right\} \epsilon_{1}^{2} \delta_{1}^{2} \\
& +\left\{-(40 / q+19) \alpha^{2}+(44 / q+62) \alpha-(104 / q+164)+(34 / 3 q+187 / 12) \pi^{2}\right\} \epsilon_{1}^{2} \delta_{2} \\
& +\left(-6 \alpha^{2}+4 \alpha+24-5 \pi^{2} / 2\right) \epsilon_{1} \delta_{1}^{3}+\left(-4 \alpha^{2}+10 \alpha-106+34 \pi^{2} / 3\right) \epsilon_{1} \delta_{1} \delta_{2} \\
& +\left(-10 \alpha^{2}+10 \alpha-22+17 \pi^{2} / 6\right) \epsilon_{1} \delta_{3}+\left(6 \alpha^{2}-24+5 \pi^{2} / 2\right) \delta_{1}^{4} \\
& +\left(-12 \alpha^{2}+40-4 \pi^{2}\right) \delta_{1}^{2} \delta_{2}+\left(4 \alpha^{2}-8+2 \pi^{2} / 3\right) \delta_{1} \delta_{3} \\
& +\left(3 \alpha^{2}-8+3 \pi^{2} / 4\right) \delta_{2}^{2}+\left(-\alpha^{2}+\pi^{2} / 12\right) \delta_{4} .
\end{aligned}
$$

\section{Appendix B: Cosmological parameters for large-field potentials}

The LF slow-roll parameters are determined by

$$
\epsilon_{1}^{q}=\frac{q p}{2} \frac{1}{X},
$$




$$
\begin{aligned}
\delta_{1}^{q} & =\frac{1}{2} \frac{(2-2 p+q p)}{X}, \\
\delta_{2}^{q} & =\frac{1}{2} \frac{(2-2 p+q p)(3-2 p+q p)}{X^{2}}, \\
\delta_{3}^{q} & =\frac{1}{4} \frac{(2-2 p+q p)(3-2 p+q p)(10-6 p+3 q p)}{X^{3}}, \\
\delta_{4}^{q} & =\frac{1}{4} \frac{(2-2 p+q p)(3-2 p+q p)(10-6 p+3 q p)(7-4 p+2 q p)}{X^{4}} .
\end{aligned}
$$

with $X \equiv[(q-1) p+2] N+\frac{q p}{2}$. Substituting these into Eqs.(18), (20) and (21), one finds the corresponding inflation parameters. The LF-power spectrum takes the form

$$
\begin{aligned}
P_{R c}^{L F} & =\left(\frac{H^{2}}{2 \pi \phi}\right)^{2}\left\{1+[(2-2 p+3 q p) \alpha-q p] \frac{1}{X}+\left[\left(\left(3 q^{2}-7 q / 2+1\right) p^{2}+(2 q-1) p\right) \alpha^{2}\right.\right. \\
& +\alpha\left(-q^{2} p^{2} / 2+q p\right)+5 \pi^{2} q^{2} p^{2} / 4-45 q^{2} p^{2} / 4-41 \pi^{2} q p / 24+11 \pi^{2} q p / 6 \\
& \left.\left.+14 q p^{2}-15 q p+7 \pi^{2} p^{2} / 12-4 p^{2}-5 \pi^{2} p / 4+8 p+2 \pi^{2} / 3-4\right] \frac{1}{X^{2}}\right\}
\end{aligned}
$$

The LF-spectral index is given by

$$
\begin{aligned}
n(k)_{s}^{L F} & =1-\{(3 q-2) p+2\} \frac{1}{X} \\
& \left.+\left\{\alpha\left(\left(3 q^{2}-5 q+2\right) p^{2}+(8 q-6) p+4\right)\right)-\left(5 q^{2} / 2-2 q\right) p^{2}-3 q p\right\} \frac{1}{X^{2}} \\
& +\left\{\left(-3 q^{3}+8 q^{2}-7 q+2\right) p^{3}-\left(14 q^{2}-24 q+7 q+10\right) p^{2}-(20 q-16) p-8\right\} \frac{\alpha^{2}}{X^{3}} \\
& +\left\{\left(13 q^{3} / 2-23 q^{2} / 2+5 q\right) p^{3}+\left(20 q^{2}-17 q\right) p^{2}+14 q p\right\} \frac{\alpha}{X^{3}} \\
& +\left\{\left(5 \pi^{2} / 2-99 / 4\right) q^{3}-\left(71 \pi^{2} / 12+105 / 2\right) q^{2}+\left(55 \pi^{2} / 12-36\right) q-7 \pi^{2} / 6+8\right\} \frac{p^{3}}{X^{3}} \\
& +\left\{\left(26 \pi^{2} / 3-157 / 2\right) q^{2}+\left(-13 \pi^{2}+102\right) q+29 \pi^{2} / 6+32\right\} \frac{p^{2}}{X^{3}} \\
& \left.+\left\{\left(-102+13 \pi^{2}\right) q-32+29 \pi^{2} / 6\right)\right\} \frac{p^{2}}{X^{3}} \\
& +\left\{\left[\left(-68+26 \pi^{2} / 3\right) q+\left(40-19 \pi^{2} / 3\right)\right] p-16+8 \pi^{2} / 3\right\} \frac{1}{X^{3}} .
\end{aligned}
$$

The first line corresponds to the leading-order calculation. Finally, the LF-running spectral index is found to be

$$
\begin{aligned}
\frac{d n_{s}^{L F, q}}{d \ln k} & =-\left\{\left(3 q^{2}-5 q+2\right) p^{2}+(8 q-6) p+4\right\} \frac{1}{X^{2}} \\
& +\left\{\left(6 q^{3}-16 q^{2}+14 q-4\right) p^{3}+\left(28 q^{2}-48 q+20\right) p^{2}+(40 q-32) p+16\right\} \frac{\alpha}{X^{3}} \\
& +\left\{\left(-13 q^{3} / 2+23 q^{2} / 2-5 q\right) p^{3}-\left(20 q^{2}-17 q\right) p^{2}-14 q p\right\} \frac{1}{X^{3}} \\
& +\left\{\begin{array}{c}
\left(-9 q^{4}+33 q^{3}-45 q^{2}+27 q-6\right) p^{4}-\left(60 q^{3}-162 q^{2}+144 q-42\right) p^{3} \\
-\left(144 q^{2}-252 q+108\right) p^{2}-(144 q-120) p-48
\end{array}\right\} \frac{\alpha^{2}}{X^{4}}
\end{aligned}
$$




$$
\begin{aligned}
& +\left\{\begin{array}{c}
\left(45 q^{4} / 2-62 q^{3}+133 q^{2} / 2-17 q\right) p^{4}+\left(113 q^{3}-204 q^{2}+91 q\right) p^{3} \\
+\left(182 q^{2}-160 q\right) p^{2}+92 q p
\end{array}\right\} \frac{\alpha}{X^{4}} \\
& +\left\{\begin{array}{c}
\left(-155 / 2+15 \pi^{2} / 2\right) q^{4}+\left(475 / 2-101 \pi^{2} / 4\right) q^{3}-\left(268-63 \pi^{2} / 2\right) q^{2} \\
+\left(132-69 \pi^{2} / 4\right) q-24+7 \pi^{2} / 2
\end{array}\right\} \frac{p^{4}}{X^{4}} \\
& +\left\{\begin{array}{c}
\left(-394+41 \pi^{2}\right) q^{3}+\left(865-201 \pi^{2} / 2\right) q^{2}-\left(618+81 \pi^{2}\right) q+144 \\
-43 \pi^{2} / 2
\end{array}\right\} \frac{p^{3}}{X^{4}} \\
& +\left\{\left(-682+78 \pi^{2}\right) q^{2}+\left(936-123 \pi^{2}\right) q-\left(312-48 \pi^{2}\right)\right\} \frac{p^{2}}{X^{4}}
\end{aligned}
$$

The first line is the leading-order calculation. 


\section{REFERENCES}

[1] L. Randall and R. Sundrum, Phys. Rev. Lett. 83, 4690 (1999) [hep-th/9906064] .

[2] P. Binetruy, C. Deffayet and D. Langlois, Nucl. Phys. B565, 269 (2000) [hepth/9905012].

[3] P. Binetruy, C. Deffayet, U. Ellwanger, and D. Langlois, Phys. Lett. B477, 285 (2000) [hep-th/9910219].

[4] H. V. Peiris, et al, Astrophys. J. Suppl. 148 (2003) 213 [astro-ph/0302225]; C. L. Bennett, et al, Astrophys. J. Suppl. 148 (2003) 1[astro-ph/0302207]; D. N. Spergel, et al, Astrophys. J. Suppl. 148 (2003) 175[astro-ph/0302209].

[5] M. Tegmark, et al [SDSS collaboration], Phys. Rev. D 69 (2004) 103501 [astro$\mathrm{ph} / 0310723]$.

[6] R. Maartens, D. Wands, B. A. Bassett, and I. P. Heard, Phys. Rev. D62 (2000) 041301 [hep-ph/9912464].

[7] A. R. Liddle and A. N. Taylor, Phys. Rev. D 65 (2002) 041301 [astro-ph/0109412].

[8] C. Charmousis and J-F. Defaux, Class. Quantum. Grav. 19 (2002) 4671 [hepth/0202107]; S. C. Davis, Phys. Rev. D 67 (2003) 024030 [hep-th/0208205]; J. E. Lidsey and N. Nunes, Phys. Rev. D 67 (2003) 103510 [asrtro-ph/0303168];

K. Maeda and T. Torii, Phys. Rev. D 69 (2004) 024002 [hep-th/0309152].

[9] E. Ramirez and A. R. Liddle, Phys. Rev. D 69 (2004) 083522 [asrtro-ph/0309608].

[10] S. Tsujikawa and A. R. Liddle, JCAP 0403 (2004) 001 [asrtro-ph/0312162].

[11] E. D. Stewart and D. H. Lyth, Phys. Lett. B302 (1993) 171.

[12] D. J. Schwarz, C. A. Terrero-Escalante, and A. A. Garcia, Phys.Lett. B 517 (2001) 243 [astro-ph/0106020].

[13] G. Calcagni, Phys. Rev. D 69 (2004) 103508 [hep-ph/0402126].

[14] E. Stewart and J.-O. Gong, Phys. Lett. B 510 (2001) 1 [astro-ph/0101225].

[15] Kyong Hee Kim, H. W. Lee, and Y. S. Myung, Phys. Rev. D 70 (2004) 027302 [hepth/0403210].

[16] G. Huey and J. E. Lidsey, Phys. Lett. B 514 (2001) 217 [astro-ph/0104006]; G. Huey and J. E. Lidsey, Phys. Rev. D 66 (2002) 043514 [astro-ph/0205236]. D. Seery and A. Taylor, astro-ph/0309512.

[17] G. Calcagni, JCAP 0311 (2003) 009 [hep-ph/0310304]; G. Calcagni, JCAP 0406 (2004) 002 [hep-ph/0312246].

[18] J. F. Dufaux, J. E. Lidsey, Maartens, and M. Sami, hep-th/0404161.

[19] S. Tsujikawa, M. Sami, and R. Maartens, astro-ph/0406078.

[20] D. Langlois, R. Maartens, and D. Wands, Phys. Lett. B 489 (2000) 259 [hepth/0006007];

[21] W. Kinney, astro-ph/0406670.

[22] V. F. Mukhanov, JETP Lett. 41 (1985) 493; V. F. Mukhanov , H. A. Feldman, and Robert H. Brandenberger Phys. Rept. 215 (1992) 203; M. Sasaki, Prog. Theor. Phys. 76 (1986) 1036.

[23] K. Koyama, D. Langlois, R. Maartens, and D. Wands, hep-th/0408222.

[24] Hungsoo Kim, G. S. Lee, and Y. S. Myung, hep-th/0402018; Y. S. Myung, hepth/0407066.

[25] Hungsoo Kim, G. S. Lee, H. W. Lee, and Y. S. Myung, Phys. Rev. D 70 (2004) 043521 [hep-th/0402198]. 
[26] Kyong Hee Kim and Y. S. Myung, astro-ph/0406387.

[27] G. Calcagni, hep-th/0406006.

[28] G. Calcagni and S. Tsujikawa, astro-ph/0407543. 